PROCEEDINGS OF THE

AMERICAN MATHEMATICAL SOCIETY

Volume 132, Number 5, Pages 1393-1401

S 0002-9939(03)07144-2

Article electronically published on December 5, 2003

\title{
ESTIMATES FOR AN OSCILLATORY INTEGRAL OPERATOR RELATED TO RESTRICTION TO SPACE CURVES
}

\author{
JONG-GUK BAK AND SANGHYUK LEE \\ (Communicated by Andreas Seeger)
}

ABstRact. We consider the oscillatory integral operator defined by

$$
T_{\lambda} f(x)=\int_{\mathbb{R}} e^{i \lambda \phi(x, t)} a(x, t) f(t) d t
$$

where $\lambda>1, a \in C_{c}^{\infty}\left(\mathbb{R}^{n} \times \mathbb{R}\right)$ and $\phi$ is a real-valued function in $C^{\infty}\left(\mathbb{R}^{n} \times \mathbb{R}\right)$. This operator may be thought of as a variable-curve version of the adjoint of the Fourier restriction operator for space curves. Under a certain nondegeneracy condition on $\phi$, we obtain $L^{p}-L^{q}$ estimates for $T_{\lambda}$ with a suitable bound for the operator norm $\left\|T_{\lambda}\right\|_{L^{p} \rightarrow L^{q}}$. This generalizes a result of Hörmander for the plane to higher dimensions.

\section{InTRODUCTION AND STATEMENT OF RESUlts}

Let $n \geq 2$ and assume that $\phi$ is a real-valued function in $C^{\infty}\left(\mathbb{R}^{n} \times \mathbb{R}\right)$, and let $a \in C_{c}^{\infty}\left(\mathbb{R}^{n} \times \mathbb{R}\right)$. Let us consider the oscillatory integral operator $T_{\lambda}$ defined by

$$
T_{\lambda} f(x)=\int_{\mathbb{R}} e^{i \lambda \phi(x, t)} a(x, t) f(t) d t
$$

for $\lambda>1$. We are interested in the problem of obtaining $L^{p}-L^{q}$ estimates for $T_{\lambda}$ with a suitable bound for the operator norm $\left\|T_{\lambda}\right\|_{L^{p} \rightarrow L^{q}}$, as $\lambda \rightarrow \infty$, with some suitable conditions imposed on the phase function $\phi$. When $n=2$, Hörmander $[\mathrm{H}$ ] (see also [S]) showed that for $1 / p+3 / q \leq 1, q>4$, there is a constant $C$ such that

$$
\left\|T_{\lambda} f\right\|_{L^{q}\left(\mathbb{R}^{2}\right)} \leq C \lambda^{-2 / q}\|f\|_{L^{p}(\mathbb{R})}
$$

provided that the phase function $\phi$ satisfies the Carleson-Sjölin condition (see [CS]), namely,

$$
\operatorname{det}\left(\partial_{t}\left(\nabla_{x} \phi\right), \partial_{t}^{2}\left(\nabla_{x} \phi\right)\right) \neq 0
$$

on the support of the cutoff function $a$. Here $\nabla_{x}$ is the gradient with respect to $x$. By rescaling and duality, (1.2) implies the sharp restriction theorem for plane curves with nonvanishing curvature (see Remark 1.2). We may think of $T_{\lambda}$ as a variablecurve version of the adjoint of the Fourier restriction operator for nondegenerate curves in $\mathbb{R}^{n}$. The purpose of this note is to obtain an extension of the estimate (1.2) to higher dimensions.

If we set $\phi(x, t)=x \cdot \gamma(t)$ and $\lambda=1$ in (1.1), then $T_{\lambda}$ is precisely the adjoint of the Fourier restriction operator for the curve $t \rightarrow \gamma(t)$. The problem of obtaining

Received by the editors October 15, 2002 and, in revised form, December 16, 2002.

2000 Mathematics Subject Classification. Primary $42 \mathrm{~B} 10$.

Key words and phrases. Oscillatory integral, restriction theorem.

Research supported in part by KOSEF grant 1999-2-102-003-5. 


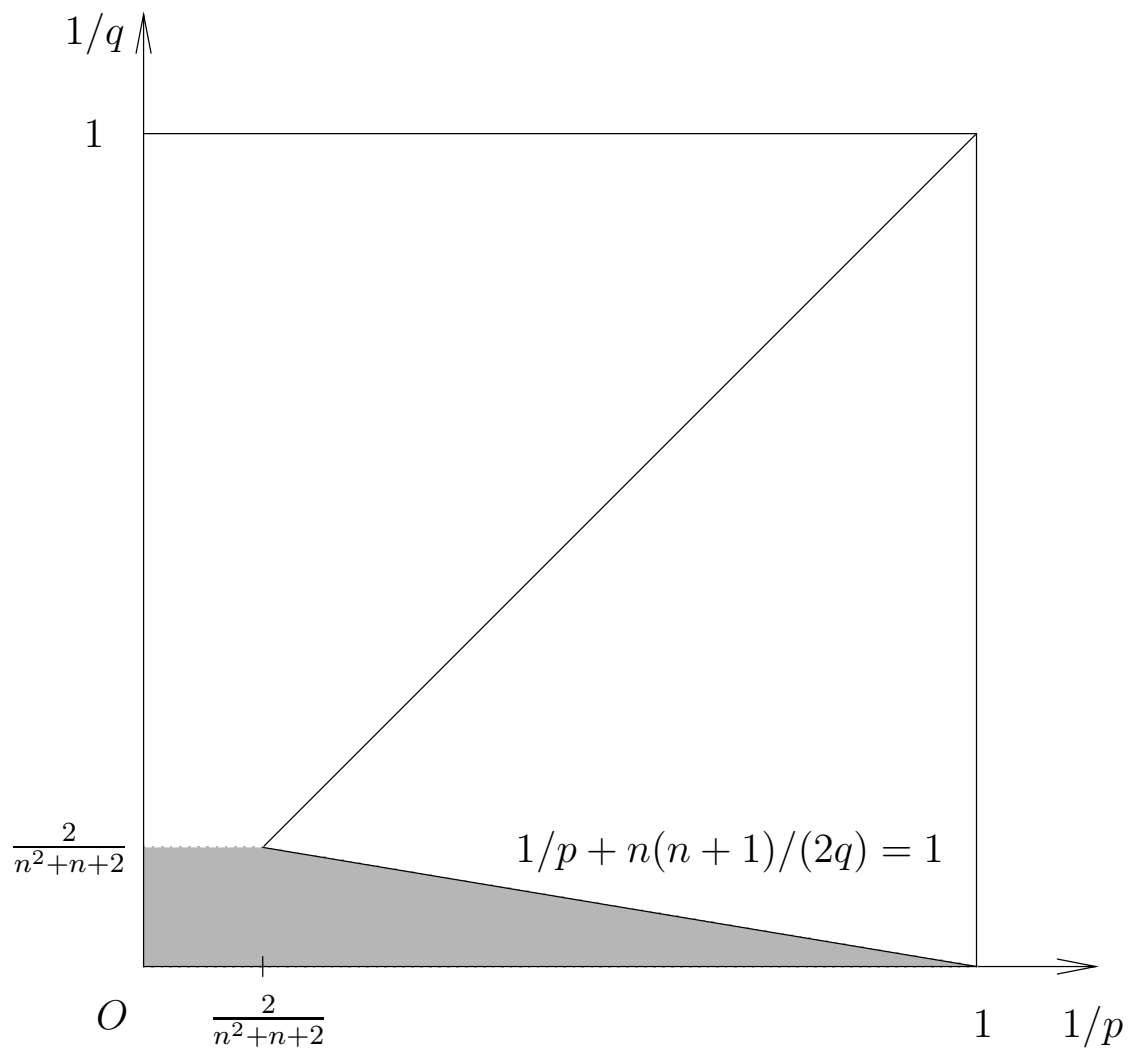

Figure 1. The known region of boundedness of $T_{\lambda}$ with $\left\|T_{\lambda}\right\|_{L^{p} \rightarrow L^{q}} \leq C \lambda^{-n / q}$

the $L^{p}-L^{q}$ estimates for the restriction of the Fourier transform to nondegenerate curves in $\mathbb{R}^{n}$ has been studied by several authors including Prestini [P1], [P2], Christ C], and Drury [D1] (see also [F], [DM2], D2, [BO]). In particular, Drury [D1] showed that the adjoint of the Fourier restriction operator for the curve $\left(t, t^{2}, \ldots, t^{n}\right)$ is bounded from $L^{p}(\mathbb{R})$ to $L^{q}\left(\mathbb{R}^{n}\right)$ for any pair $(p, q)$ satisfying $1 / p+n(n+1) /(2 q) \leq$ $1, q>\left(n^{2}+n+2\right) / 2$. By rescaling this result, which corresponds to the case $\phi(x, t)=x \cdot\left(t, t^{2}, \ldots, t^{n}\right)$ and $\lambda=1$ in $(1.1)$, we get $\left\|T_{\lambda}\right\|_{L^{p} \rightarrow L^{q}}=O\left(\lambda^{-n / q}\right)$ provided that $1 / p+n(n+1) /(2 q) \leq 1, q>\left(n^{2}+n+2\right) / 2$. (See Figure 1.) So it seems to be reasonable to expect that a similar estimate holds for $T_{\lambda}$ when $\phi$ is a more general phase function satisfying suitable conditions. In view of the adjoint of the restriction operator for space curves, a natural nondegeneracy condition for the phase function might be that, for each fixed $x$, the curve $t \rightarrow \nabla_{x} \phi(x, t)$ has nonvanishing torsion, that is,

$$
\operatorname{det}\left(\partial_{t}\left(\nabla_{x} \phi\right), \partial_{t}^{2}\left(\nabla_{x} \phi\right), \ldots, \partial_{t}^{n}\left(\nabla_{x} \phi\right)\right) \neq 0
$$

on the support of the cutoff function $a$.

Our result may be stated as follows.

Theorem 1.1. Let $T_{\lambda}$ be given by (1.1). Suppose that $\phi$ satisfies (1.3) on the support of a. If $1 / p+n(n+1) /(2 q) \leq 1, q>\left(n^{2}+n+2\right) / 2$, then there is a 
constant $C$, independent of $f$ and $\lambda>1$, such that

$$
\left\|T_{\lambda} f\right\|_{L^{q}\left(\mathbb{R}^{n}\right)} \leq C \lambda^{-n / q}\|f\|_{L^{p}(\mathbb{R})} .
$$

Remark 1.2. The condition $1 / p+n(n+1) /(2 q) \leq 1$ is necessary for (1.4) to hold. To see this, just replace $f$ by $e^{-i \lambda \phi\left(x_{0}, t\right)} f$, and replace $x$ by $x_{0}+x / \lambda$ in (1.1). Then (1.4) implies that

$$
\left\|\int_{\mathbb{R}} e^{i \lambda\left(\phi\left(x_{0}+x / \lambda, t\right)-\phi\left(x_{0}, t\right)\right)} a\left(x_{0}+x / \lambda, t\right) f(t) d t\right\|_{q} \leq C\|f\|_{p} .
$$

Letting $\lambda \rightarrow \infty$, we get

$$
\left\|\int_{\mathbb{R}} e^{i x \cdot \nabla_{x} \phi\left(x_{0}, t\right)} a\left(x_{0}, t\right) f(t) d t\right\|_{q} \leq C\|f\|_{p}
$$

by Fatou's lemma and the Dominated Convergence Theorem. By adapting the proof of the necessary conditions for the $L^{p}-L^{q}$ estimates for the restriction to nondegenerate curves (see [P2]), we may see that (1.5) cannot hold unless $1 / p+$ $n(n+1) /(2 q) \leq 1$. More precisely, let us assume that $a\left(x_{0}, t_{0}\right)>0$ and apply Taylor's theorem to each component of $\nabla_{x} \phi\left(x_{0}, t\right)$ to get

$$
\begin{aligned}
\nabla_{x} \phi\left(x_{0}, t\right)= & \nabla_{x} \phi\left(x_{0}, t_{0}\right)+\partial_{t}\left(\nabla_{x} \phi\right)\left(x_{0}, t_{0}\right)\left(t-t_{0}\right)+\partial_{t}^{2}\left(\nabla_{x} \phi\right)\left(x_{0}, t_{0}\right)\left(t-t_{0}\right)^{2} / 2 ! \\
& +\cdots+\partial_{t}^{n}\left(\nabla_{x} \phi\right)\left(x_{0}, t_{0}\right)\left(t-t_{0}\right)^{n} / n !+E(t)
\end{aligned}
$$

where each component of $E(t)$ is $O\left(\left|t-t_{0}\right|^{n+1}\right)$ near $t_{0}$. So

$$
\left|x \cdot\left(\nabla_{x} \phi\left(x_{0}, t\right)-\nabla_{x} \phi\left(x_{0}, t_{0}\right)\right)\right|<1
$$

if $\left|t-t_{0}\right|<c \delta$ for $c>0$ sufficiently small and if $\left|x \cdot u_{j}\right|<\delta^{-j}, 1 \leq j \leq n$, where $u_{j}=\partial_{t}^{j}\left(\nabla_{x} \phi\right)\left(x_{0}, t_{0}\right) / j$ ! . Thus, if we take $f$ to be the characteristic function of the interval $\left(t_{0}, t_{0}+c \delta\right)$ for $\delta$ small, then the integral on the left-hand side of (1.5) is bounded below in absolute value by a multiple of $\delta$ on the parallelepiped given by $\left|x \cdot u_{j}\right|<\delta^{-j}, 1 \leq j \leq n$. Since (1.3) holds, this parallelepiped is nondegenerate and it has volume comparable to $\delta^{-n(n+1) / 2}$. So (1.5) implies that

$$
\delta^{1-n(n+1) / 2 q} \leq C \delta^{1 / p}
$$

for every small $\delta>0$. Therefore, we must have $1 / p+n(n+1) /(2 q) \leq 1$ if (1.4) holds.

It seems to be a lot harder to prove that the condition $q>\left(n^{2}+n+2\right) / 2$ is necessary for (1.4) and (1.5), although there appears to be some evidence that this is the case. In the special case of $\phi(x, t)=x \cdot\left(t, t^{2}, \ldots, t^{n}\right)$, the condition $q>\left(n^{2}+n+2\right) / 2$ is known to be necessary for (1.5) for $n=2,3$ (see [M]). When $n \geq 4$, for the same choice of $\phi$, Mockenhaupt [M] showed the weaker necessary condition that if (1.5) holds for some $p$, then $q \geq\left(n^{2}+n+2\right) / 2$.

The estimate (1.4) remains valid under small smooth perturbations of the phase function $\phi$ and the amplitude $a$. This stability (or uniformity) plays an important role in the proof of our result. The proof is essentially an adaptation of an argument of Drury [D1, but we also use some methods of Hörmander and Bourgain. It is an inductive argument based on the fact that a known $L^{p}-L^{q}$ estimate for $T_{\lambda}$ for a pair $(1 / p, 1 / q)$ on the critical line, i.e., satisfying $1 / p+n(n+1) /(2 q)=1$, can be combined with a related $L^{2}$ estimate to obtain estimates for a larger range of pairs of exponents on the critical line. In this way we can obtain all estimates for $T_{\lambda}$ on 
the half-open segment $1 / p+n(n+1) /(2 q)=1,0 \leq 1 / q<2 /\left(n^{2}+n+2\right)$ in the $(1 / p, 1 / q)$-plane.

Remark 1.3. A geometric formulation of the Carleson-Sjölin condition is contained in a paper by Mockenhaupt, Seeger and Sogge [MSS. The hypothesis (1.3) is a natural generalization of that condition. A related curvature condition appeared also in a paper by Greenleaf and Seeger [GS]. Our assumption that the curve $t \rightarrow \phi_{x}^{\prime}(x, t)=\nabla_{x} \phi(x, t)$ has nonvanishing torsion for every $(x, t)$ is invariant under the changes of variables $x=x(u), t=t(s)$ when these functions are diffeomorphisms. Indeed these transformations induce linear changes of variables in the fibers of the canonical relation $\left\{\left(x, \phi_{x}^{\prime}, t,-\phi_{t}^{\prime}\right)\right\}$, and the torsion condition is clearly invariant under linear changes of variables. (The latter assertion may also be checked directly.)

We would like to thank the referee for pointing out the connections between the condition (1.3) and some ideas in the works just mentioned. We thank also Andreas Seeger for very helpful conversations on these matters.

\section{Preliminaries}

Before beginning the proof we state three well-known lemmas which will be used later. A function $F$ on $\mathbb{R}^{n}$ is called symmetric if $F\left(x_{1}, \ldots, x_{n}\right)=F\left(x_{\sigma(1)}, \ldots, x_{\sigma(n)}\right)$ for any permutation $\sigma$ on $n$ letters. Let $g_{1}, \ldots, g_{n}$ be the elementary symmetric polynomials in $n$ variables, that is,

$$
\begin{aligned}
g_{1}\left(x_{1}, \ldots, x_{n}\right) & =x_{1}+\cdots+x_{n}, \\
g_{2}\left(x_{1}, \ldots, x_{n}\right) & =x_{1} x_{2}+\cdots+x_{1} x_{n}+x_{2} x_{3}+\cdots+x_{2} x_{n}+\cdots+x_{n-1} x_{n}, \\
\vdots & \\
g_{n}\left(x_{1}, \ldots, x_{n}\right) & =x_{1} x_{2} \cdots x_{n} .
\end{aligned}
$$

The following result may be found in Glaeser $[\mathrm{G}]$. (See also $[\mathrm{GG}]$.)

Lemma 2.1. If $F$ is a smooth symmetric function on $\mathbb{R}^{n}$, then there is a smooth function $H: \mathbb{R}^{n} \rightarrow \mathbb{R}$ satisfying $F(x)=H\left(g_{1}(x), \ldots, g_{n}(x)\right)$.

The next lemma is just a restatement of Lemma 1 in [DM1].

Lemma 2.2. Let $v(h)=v\left(h_{1}, \ldots, h_{n-1}\right)=\left|h_{1} \cdots h_{n-1}\right| \prod_{1 \leq i<j \leq n-1}\left|h_{i}-h_{j}\right|$. Then for $\lambda>0$,

$$
\left|\left\{h \in \mathbb{R}^{n-1}: v(h) \leq \lambda\right\}\right| \leq C \lambda^{2 / n} .
$$

Proof. We just note that, by homogeneity, it suffices to show that

$$
\left|\left\{h \in \mathbb{R}^{n-1}: v(h) \leq 1\right\}\right| \leq C .
$$

For the proof of this fact the reader is referred to [DM1].

Let $L^{p, q}$ denote the Lorentz space with the (quasi-)norm $\|\cdot\|_{p, q}$. (See e.g. [BL].) The following result on interpolation is implicit in $[\mathrm{B}$. A general version may be found in [CSWW].

Lemma 2.3. Let $\varepsilon_{0}, \varepsilon_{1}>0$. Suppose that $T_{j}$ is a sequence of linear operators such that $\left\|T_{j} f\right\|_{q_{0}, \infty} \leq M_{0} 2^{-\varepsilon_{0} j}\|f\|_{p_{0}, 1}$ and $\left\|T_{j} f\right\|_{q_{1}, \infty} \leq M_{1} 2^{\varepsilon_{1} j}\|f\|_{p_{1}, 1}$, where 
$1 \leq p_{0}, p_{1} \leq \infty$ and $1<q_{0}, q_{1} \leq \infty$. Then $T=\sum_{j=-\infty}^{\infty} T_{j}$ is bounded from $L^{p, 1}$ to $L^{q, \infty}$ with

$$
\|T f\|_{q, \infty} \leq C M_{0}^{1-\theta} M_{1}^{\theta}\|f\|_{p, 1}
$$

where $\theta=\varepsilon_{0} /\left(\varepsilon_{0}+\varepsilon_{1}\right), 1 / q=(1-\theta) / q_{0}+\theta / q_{1}$ and $1 / p=(1-\theta) / p_{0}+\theta / p_{1}$.

\section{Proof of Theorem 1.1}

We will deduce Theorem 1.1 from the following result.

Proposition 3.1. Let $n \geq 3$. Suppose that for some $r, s \in[1, \infty]$ satisfying $1 / r+$ $n(n+1) /(2 s)=1, s>\left(n^{2}+n+2\right) / 2$, the estimate

$$
\left\|T_{\lambda} f\right\|_{L^{s, \infty}\left(\mathbb{R}^{n}\right)} \leq C \lambda^{-n / s}\|f\|_{L^{r, 1}(\mathbb{R})}
$$

holds, and suppose also that this estimate holds with the same constant $C$ under small smooth perturbations of $\phi$ and a. If $\phi$ satisfies (1.3), then

$$
\left\|T_{\lambda} f\right\|_{L^{q, \infty}\left(\mathbb{R}^{n}\right)} \leq C \lambda^{-n / q}\|f\|_{L^{p, 1}(\mathbb{R})}
$$

holds for $1 / p+n(n+1) /(2 q)=1,1 / q=(n-2) /[n(n+2) s]+2 /[n(n+2)]$. Here the new constant $C$ is again stable under small smooth perturbations of $\phi$ and $a$.

Proof of Theorem 1.1. It is easy to deduce Theorem 1.1 from Proposition 3.1 by using induction. We begin induction by applying Proposition 3.1 to the trivial estimate $\left\|T_{\lambda} f\right\|_{\infty} \leq C\|f\|_{1}$. Obviously this constant $C$ is stable under small smooth perturbations of $\phi$ and $a$. Applying Proposition 3.1 repeatedly yields a sequence of points $\left(1 / p_{i}, 1 / q_{i}\right)$ for which (3.2) holds, which are recursively given by the relations $\left(1 / p_{0}, 1 / q_{0}\right)=(1,0)$ and

$$
\frac{1}{p_{i}}+\frac{n(n+1)}{2 q_{i}}=1, \quad \frac{1}{q_{i+1}}=\frac{(n-2)}{n(n+2) q_{i}}+\frac{2}{n(n+2)}, \quad i \geq 0 .
$$

Since the desired strong-type estimates can be obtained by real interpolation from these restricted weak-type estimates, we only need to observe that

$$
q_{i} \rightarrow\left(n^{2}+n+2\right) / 2
$$

as $i \rightarrow \infty$. But this is easy to see by the monotonicity of $q_{i}$. (Note that, given any point $\left(p_{*}, q_{*}\right)$ on the critical line, the strong-type estimate at this point can be obtained after a finite number of applications of Proposition 3.1.) Thus we have established Theorem 1.1, assuming Proposition 3.1.

Proof of Proposition 3.1. The support of $a$ may be assumed to be as small as we like, since $T_{\lambda}$ can be written as a finite sum of operators with small support by using a partition of unity. Let us consider an $n$-linear operator $L_{\lambda}$ defined by

$$
\begin{aligned}
L_{\lambda}\left(f_{1}, f_{2}, \ldots, f_{n}\right)(x) & =\prod_{i=1}^{n} T_{\lambda} f_{i}(x) \\
& =\int_{\mathbb{R}^{n}} e^{i \lambda\left(\phi\left(x, t_{1}\right)+\cdots+\phi\left(x, t_{n}\right)\right)} \prod_{i=1}^{n}\left[a\left(x, t_{i}\right) f_{i}\left(t_{i}\right)\right] d t_{1} \ldots d t_{n} .
\end{aligned}
$$

The singularities of this oscillatory integral appear on the set $\left\{t \in \mathbb{R}^{n}: \prod_{i<j} \mid t_{i}-\right.$ $\left.t_{j} \mid=0\right\}$, where $\operatorname{det} \frac{\partial^{2}}{\partial x_{i} \partial t_{j}}\left(\sum_{\ell} \phi\left(x, t_{\ell}\right)\right)$ vanishes. So we decompose $L_{\lambda}$ dyadically 
away from this set. Thus, for any integer $k$, put

$$
S_{k}=\left\{t \in \mathbb{R}^{n}: 2^{-k-1} \leq \prod_{1 \leq i<j \leq n}\left|t_{i}-t_{j}\right|<2^{-k}\right\}
$$

and $\chi_{k}=\chi_{S_{k}}$. Now let

$$
\begin{aligned}
& L_{\lambda}^{k}\left(f_{1}, f_{2}, \ldots, f_{n}\right)(x) \\
& =\int_{\mathbb{R}^{n}} e^{i \lambda\left(\phi\left(x, t_{1}\right)+\cdots+\phi\left(x, t_{n}\right)\right)} \chi_{k}\left(t_{1}, \ldots, t_{n}\right) \prod_{i=1}^{n}\left[a\left(x, t_{i}\right) f_{i}\left(t_{i}\right)\right] d t_{1} \ldots d t_{n} .
\end{aligned}
$$

An $L^{2}$ estimate for $L_{\lambda}^{k}$ : We write $s=\left(s_{1}, s_{2}, \ldots, s_{n}\right)$ and $t=\left(t_{1}, \ldots, t_{n}\right)$. Let us make the change of variables given by

$$
s=G(t)=\left(g_{1}\left(t_{1}, \ldots, t_{n}\right), g_{2}\left(t_{1}, \ldots, t_{n}\right), \ldots, g_{n}\left(t_{1}, \ldots, t_{n}\right)\right)
$$

where the $g_{j}$ are the elementary symmetric polynomials mentioned in Section 2 . Since the mapping $t \rightarrow s$ is defined by polynomials of degree at most $n$, Bézout's theorem implies that the multiplicity of the mapping $t \rightarrow s$ is at most $n$ ! except on a set of measure zero. This means that we can decompose $\mathbb{R}^{n}$ (except a set of measure zero) into a finite number of sets $\Omega_{1}, \ldots, \Omega_{M}$ so that on each $\Omega_{i}$ the map $t \rightarrow s$ is one-to-one. So it follows that

$$
\begin{aligned}
L_{\lambda}^{k} & \left(f_{1}, f_{2}, \ldots, f_{n}\right)(x) \\
\quad & =\sum_{j=1}^{M} \int_{G\left(\Omega_{j}\right)} e^{i \lambda \Phi(x, s)} \chi_{k}(t(s)) A(x, s) \prod_{i=1}^{n} f_{i}\left(t_{i}(s)\right)\left|\frac{\partial t}{\partial s}\right| d s
\end{aligned}
$$

where

$$
\Phi(x, s)=\phi\left(x, t_{1}(s)\right)+\cdots+\phi\left(x, t_{n}(s)\right) \text { and } A(x, s)=\prod_{i=1}^{n} a\left(x, t_{i}(s)\right) .
$$

The condition (1.3) implies that if the support of $a$ is sufficiently small, then there is a constant $c>0$ such that

$$
\left|\operatorname{det}\left(\frac{\partial^{2} \Phi(x, s)}{\partial x_{i} \partial s_{j}}\right)\right| \geq c
$$

for all $(x, s) \in \operatorname{supp} A$.

We may see this as follows. A simple computation shows that

$$
\left|\frac{\partial s}{\partial t}\right|=\left|\frac{\partial\left(s_{1} \cdots s_{n}\right)}{\partial\left(t_{1} \cdots t_{n}\right)}\right|=\prod_{1 \leq i<j \leq n}\left|t_{i}-t_{j}\right| .
$$

Now set

$$
\gamma_{x}(t)=\nabla_{x} \phi(x, t)
$$

By a generalization of the mean value theorem (see [PS], part $V$, Chapter 1, problem 95), there exist $u_{1}, \ldots, u_{n} \in\left(\min _{i} t_{i}(s), \max _{i} t_{i}(s)\right)$ such that

$$
\operatorname{det}\left(\gamma_{x}^{\prime}\left(t_{1}(s)\right), \cdots, \gamma_{x}^{\prime}\left(t_{n}(s)\right)\right)=\frac{1}{c_{n}} \operatorname{det}\left(\gamma_{x}^{\prime}\left(u_{1}\right), \ldots, \gamma_{x}^{(n)}\left(u_{n}\right)\right) \prod_{i<j}\left|t_{i}(s)-t_{j}(s)\right|
$$


where $c_{n}=2 ! 3 ! \cdots(n-1)$ !. So (3.6) follows from (1.3), since

$$
\begin{aligned}
\left|\operatorname{det}\left(\frac{\partial^{2} \Phi(x, s)}{\partial x_{i} \partial s_{j}}\right)\right| & =\left|\operatorname{det}\left(\gamma_{x}^{\prime}\left(t_{1}(s)\right), \ldots, \gamma_{x}^{\prime}\left(t_{n}(s)\right)\right)\right|\left|\frac{\partial t}{\partial s}\right| \\
& =\frac{1}{2}\left|\operatorname{det}\left(\gamma_{x}^{\prime}\left(u_{1}\right), \ldots, \gamma_{x}^{(n)}\left(u_{n}\right)\right)\right| .
\end{aligned}
$$

Lemma 2.1 implies that $\Phi$ and $A$ are smooth functions with respect to $s$. This fact and (3.6) allow us to apply a well-known oscillatory integral estimate of Hörmander (see [S], p. 377) to (3.5). Thus we obtain

$$
\left\|L_{\lambda}^{k}\left(f_{1}, f_{2}, \ldots, f_{n}\right)\right\|_{2}^{2} \leq C \lambda^{-n} \int \chi_{k}(t(s)) \prod_{i=1}^{n}\left|f_{i}\left(t_{i}(s)\right)\right|^{2}\left|\frac{\partial t}{\partial s}\right|^{2} d s .
$$

By reversing the change of variables made above, we get

$$
\left\|L_{\lambda}^{k}\left(f_{1}, f_{2}, \ldots, f_{n}\right)\right\|_{2}^{2} \leq C \lambda^{-n} \int \chi_{k}(t) \prod_{i=1}^{n}\left|f_{i}\left(t_{i}\right)\right|^{2} \prod_{1 \leq i<j \leq n}\left|t_{i}-t_{j}\right|^{-1} d t .
$$

Since

$$
\sup _{t_{1}}\left|\left\{\left(t_{2}, \ldots, t_{n}\right): \prod_{1 \leq i<j \leq n}\left|t_{i}-t_{j}\right| \sim 2^{-k}\right\}\right|=\left|\left\{h \in \mathbb{R}^{n-1}: v(h) \sim 2^{-k}\right\}\right|,
$$

it follows that

$$
\left\|L_{\lambda}^{k}\left(f_{1}, f_{2}, \ldots, f_{n}\right)\right\|_{2} \leq C \lambda^{-n / 2} 2^{k / 2}\left|\left\{h \in \mathbb{R}^{n-1}: v(h) \leq 2^{-k}\right\}\right|^{1 / 2}\left\|f_{1}\right\|_{2} \prod_{i=2}^{n}\left\|f_{i}\right\|_{\infty} .
$$

Hence, by Lemma 2.2, we have

$$
\left\|L_{\lambda}^{k}\left(f_{1}, f_{2}, \ldots, f_{n}\right)\right\|_{2} \leq C \lambda^{-n / 2} 2^{(n-2) k / 2 n}\left\|f_{1}\right\|_{2} \prod_{i=2}^{n}\left\|f_{i}\right\|_{\infty} .
$$

An estimate following from the hypothesis (3.1): For $h=\left(h_{1}, \ldots, h_{n-1}\right) \in$ $\mathbb{R}^{n-1}$, define $\phi_{h}$ by

$$
\phi_{h}(x, t)=\phi(x, t)+\phi\left(x, t+h_{1}\right)+\cdots+\phi\left(x, t+h_{n-1}\right) .
$$

We make the change of variables $t_{1} \rightarrow \tau, t_{2} \rightarrow \tau+h_{1}, \ldots, t_{n} \rightarrow \tau+h_{n-1}$ in (3.4) to get

$$
L_{\lambda}^{k}\left(f_{1}, f_{2}, \ldots, f_{n}\right)(x)=\int_{\left\{h \in \mathbb{R}^{n-1}: v(h) \sim 2^{-k}\right\}} \int e^{i \lambda \phi_{h}(x, \tau)} a_{h}(x, \tau) f_{h}(\tau) d \tau d h
$$

where

$$
a_{h}(x, \tau)=a(x, \tau) \prod_{i=2}^{n} a\left(x, \tau+h_{i-1}\right) \text { and } f_{h}(\tau)=f_{1}(\tau) \prod_{i=2}^{n} f_{i}\left(\tau+h_{i-1}\right) .
$$

For $h$ and $x$ fixed let

$$
\gamma_{x}^{h}(t)=\nabla_{x} \phi_{h}(x, t) .
$$

From the smoothness of $\phi$, it follows immediately by continuity that if $|h|$ is sufficiently small, then $\frac{1}{n}\left(\frac{d}{d \tau}\right)^{k} \gamma_{x}^{h}(\tau)$ is close to $\left(\frac{d}{d \tau}\right)^{k} \gamma_{x}(\tau)$. Therefore, if the support of $a$ is sufficiently small, then the linear independence of

$$
\frac{d}{d \tau} \gamma_{x}^{h}(\tau), \ldots,\left(\frac{d}{d \tau}\right)^{n} \gamma_{x}^{h}(\tau)
$$


is a consequence of the linear independence of $\gamma_{x}^{\prime}(\tau), \gamma_{x}^{\prime \prime}(\tau), \ldots, \gamma_{x}^{(n)}(\tau)$, which is the condition (1.3). Thus the phase function $\phi_{h}$ satisfies the assumption (1.3) uniformly in small $h$. Therefore, after a use of Minkowski's inequality we may apply the estimate in (3.1) to the inner integral in (3.9) with a bound uniform in small $h$. This yields

$$
\left\|L_{\lambda}^{k}\left(f_{1}, f_{2}, \ldots, f_{n}\right)\right\|_{s, \infty} \leq C \lambda^{-n / s}\left|\left\{h \in \mathbb{R}^{n-1}: v(h) \leq 2^{-k}\right\}\right| \cdot\left\|f_{1}\right\|_{r, 1} \prod_{i=2}^{n}\left\|f_{i}\right\|_{\infty} .
$$

Using Lemma 2.2 again, we obtain

$$
\left\|L_{\lambda}^{k}\left(f_{1}, f_{2}, \ldots, f_{n}\right)\right\|_{s, \infty} \leq C \lambda^{-n / s} 2^{-2 k / n}\left\|f_{1}\right\|_{r, 1} \prod_{i=2}^{n}\left\|f_{i}\right\|_{\infty} .
$$

Summation of the estimates for $L_{\lambda}^{k}$ : We have $L_{\lambda}=\sum_{k=-\infty}^{\infty} L_{\lambda}^{k}$. Let us fix $f_{2}, \ldots, f_{n} \in L^{\infty}$ and apply Lemma 2.3 to (3.8) and (3.10). This gives

$$
\left\|L_{\lambda}\left(f_{1}, f_{2}, \ldots, f_{n}\right)\right\|_{b, \infty} \leq C \lambda^{-n / b}\left\|f_{1}\right\|_{a, 1} \prod_{i=2}^{n}\left\|f_{i}\right\|_{\infty}
$$

where

$$
\frac{1}{a}=\frac{n-2}{n+2}\left(\frac{1}{r}\right)+\frac{2}{n+2}, \quad \frac{1}{b}=\frac{n-2}{n+2}\left(\frac{1}{s}\right)+\frac{2}{n+2} .
$$

Interpolating the $n$ estimates obtained by permuting the functions in (3.11), and then setting each $f_{j}=f$, we get

$$
\left\|T_{\lambda} f\right\|_{q, \infty} \leq C \lambda^{-n / q}\|f\|_{p, 1}
$$

where $p=n a$ and $q=n b$. The constant $C$ is stable under small smooth perturbations of $\phi$ and $a$, since the same holds for the constants $C$ in (3.8) and (3.10). From the fact that $1 / r+n(n+1) /(2 s)=1$, it follows that

$$
\frac{1}{p}+\frac{n(n+1)}{2 q}=1
$$

Note also that $q$ and $s$ are related by

$$
\frac{1}{q}=\frac{(n-2)}{n(n+2) s}+\frac{2}{n(n+2)}
$$

This completes the proof of Proposition 3.1.

\section{REFERENCES}

[BO] J.-G. Bak and D. Oberlin, A note on Fourier restriction for curves in $\mathbb{R}^{3}$, Proceedings of the AMS Conference on Harmonic Analysis, Mt. Holyoke College (June 2001), Contemp. Math., Vol. 320, Amer. Math. Soc., Providence, RI, 2003.

[B] J. Bourgain, Estimations de certaines fonctions maximales, C. R. Acad. Sci. Paris 301 (1985), 499-502. MR 87b:42023

[BL] J. Bergh and J. Löfström, Interpolation spaces. An introduction, Springer-Verlag, New York, 1976. MR 58:2349

[C] M. Christ, On the restriction of the Fourier transform to curves: endpoint results and the degenerate case, Trans. Amer. Math. Soc. 287 (1985), 223-238. MR 87b:42018

[CS] L. Carleson and P. Sjölin, Oscillatory integrals and a multiplier problem for the disk, Studia Math. 44 (1972), 287-299. MR 50:14052

[CSWW] A. Carbery, A. Seeger, S. Wainger, and J. Wright, Classes of singular integral operators along variable lines, J. Geom. Anal. 9 (1999), 583-605. MR 2001g:42026 
[D1] S. Drury, Restrictions of Fourier transforms to curves, Ann. Inst. Fourier (Grenoble) 35 (1985), 117-123. MR 86e:42026

[D2] S. Drury, Degenerate curves and harmonic analysis, Math. Proc. Cambridge Philos. Soc. 108 (1990), 89-96. MR 91h:42021

[DM1] S. Drury and B. Marshall, Fourier restriction theorems for curves with affine and Euclidean arclengths, Math. Proc. Cambridge Philos. Soc. 97 (1985), 111-125. MR 87b:42019

[DM2] S. Drury and B. Marshall, Fourier restriction theorems for degenerate curves, Math. Proc. Cambridge Philos. Soc. 101 (1987), 541-553. MR 88e:42026

[F] C. Feffermann, Inequalities for strongly singular convolution operators, Acta Math. 124 (1970), 9-36. MR 41:2468

[G] G. Glaeser. Fonctions composées différentiables, Ann. of Math. 77 (1963), 193-209. MR 26:624

[GG] M. Golubitsky and V. Guillemin, Stable mappings and their singularities, Graduate Texts in Mathematics, Vol. 14, Springer-Verlag, New York-Heidelberg, 1973. MR 49:6269

[GS] A. Greenleaf and A. Seeger, Fourier integral operators with cusp singularities, Amer. J. Math. 120 (1998), 1077-1119. MR 99g:58120

$[\mathrm{H}] \quad$ L. Hörmander, Oscillatory integrals and multipliers on $F L^{p}$, Ark. Mat. 11 (1973), 1-11. MR 49:5674

[M] G. Mockenhaupt, Bounds in Lebesgue spaces of oscillatory integral operators, Habilitationsschrift, Universität Siegen (1996).

[MSS] G. Mockenhaupt, A. Seeger, and C. Sogge, Local smoothing of Fourier integral operators and Carleson-Sjölin estimates, J. Amer. Math. Soc. 6 (1993), 60-130. MR 93h:58150

[PS] G. Polya and G. Szegö, Problems and theorems in analysis, Die Grundlehren der mathematischen Wissenschaften, Band 216, Springer-Verlag, New York-Heidelberg, 1976. MR 53:2

[P1] E. Prestini, A restriction theorem for space curves, Proc. Amer. Math. Soc. 70 (1978), 8-10. MR 57:7026

[P2] E. Prestini, Restriction theorems for the Fourier transform to some manifolds in $R^{n}$, Proc. Sympos. Pure Math. 35 (1979), 101-109. MR 81d:42028

[S] E. M. Stein, Harmonic analysis: real-variable methods, orthogonality, and oscillatory integrals, Princeton University Press, Princeton, NJ, 1993. MR 95c:42002]

Pohang University of Science and Technology and The Korea Institute for AdVANCED STUDY

E-mail address: bak@postech.ac.kr

Pohang University of Science and Technology, Pohang 790-784, Korea

E-mail address: huk@euclid.postech.ac.kr 